\title{
Experiments and Models of Avalanches in Martensites
}

\author{
J. Ortín, I. Ràfols, Ll. Carrillo, J. Goicoechea, E. Vives, Ll. Mañosa and A. Planes \\ Departament ECM, Facultat de Física, Universitat de Barcelona, Diagonal 647, 08028 Barcelona, \\ Catalonia, Spain
}

\begin{abstract}
We have studied the kinetics of thermoelastic martensitic transformations (MT), both experimentally and using spin models. Measurements of acoustic emission and thermal pulses in $\mathrm{Cu}-\mathrm{Zn}-\mathrm{Al}$ single crystals, during thermally and stress induced $\mathrm{MT}$, show that the transformation takes place as a sequence of avalanches, whose sizes and durations distribute according to power laws in more than one decade. Most features observed are qualitatively reproduced by spin models that incorporate domain interactions and frozen disorder. A systematic simulation of these models at $T=0$ (no fluctuations allowed) has shown that there is a critical concentration of disorder for which avalanche sizes and durations distribute following power-laws, which appear to be independent of details of the models other than their dimensionality. We discuss the connection between the experimental results and the simulations.
\end{abstract}

\section{INTRODUCTION}

Extended dissipative systems with many degrees of freedom, driven by an external field, are often observed to evolve with no characteristic length and time scales. Passage from metastable to metastable state takes place by avalanches whose size and duration distribute statistically according to power-laws extending through several decades. The frequent occurence of fractals in nature and the ubiquituous presence of $1 / f$ noise in the frequency spectrum of many physical systems are thought to be signatures of such a behaviour. The origin of this remarkable dynamics has received renewed in terest in the last years, after Bak et al. introduced the possibility that extended dynamical systems could self-organize spontaneously (without having to tune a particular parameter) into a statistically stationary critical state [1]. From then on, several models exhibiting self-organized criticality (SOC) have been formulated and analyzed both numerically and analytically, and alternative mechanisms leading to power-laws have been proposed [2]. At the same time, many real extended dissipative systems (including sandpiles, earthquakes, volcanic rocks, fractured systems, and ferromagnets and other hysteretic systems) have been interrogated experimentally searching for power-laws and SOC behaviour [3].

In this context, athermal (i.e. fluctuationless) martensitic transformations (MT) can be regarded as candidates to display a dynamic behaviour with no characteristic length and time scales. Indeed, the interplay between the elastic strain energy (originating from shape and volume changes in the transformation and from lattice defects) and the external driving field (temperature or applied stress) gives rise to an evolution by avalanches [4]. The avalanches connect metastable states characterized by thermoelastic equilibrium at multiple interfaces. In addition, microstructural observations of thermoelastic martensites reveal that domains of many different sizes are formed in the MT, and the multiplate arrangement is often reminiscent of a selfsimilar geometry. 
This work focuses on the statistical properties of the dynamics of thermoelastic MT, both experimentally and through numerical simulations of spin models.

\section{EXPERIMENTS AND RESULTS}

We have carried out two different kinds of experiments.

First, we have studied the stress-induced $\mathrm{MT}$ of a $\mathrm{Cu}-19.4 \% \mathrm{Zn}-13.1$ at $\% \mathrm{Al}$ single crystal at room temperature. The crystal has a tensile shape with an average cross-section of $1.80 \mathrm{~mm}^{2}$, and its $M_{s} \sim 263 \mathrm{~K}$. It has been subjected to a uniaxial stress along the tensile axis in an infinitely-soft tensile machine driven by a dead load that varies in a range of about $8.00 \mathrm{~kg}$ at typical rates of $0.075 \mathrm{~kg} / \mathrm{min}$. The transformation events have been detected as short heat pulses, originating from the transformation latent heat, using two small thermoelectric batteries $\left(11.4 \times 9.0 \mathrm{~mm}^{2}\right)$ in series, one attached to each side of the specimen. The output of the transducers is sampled at $20 \mathrm{~Hz}$, filtered from the main time constant of the experimental set-up (centered around 4.6s), and analyzed for the amplitude and duration of heat-flow pulses larger than a given threshold. Figure 1 shows the statistical distribution of amplitudes and durations for typical stress excursions between $51.5 M P a$ ( $\beta$-phase single crystal) and 87.1MPa (two-phase region). Both magnitudes distribute in the form of power-laws $p(A) \sim A^{-\alpha}$ and $p(T) \sim T^{-r}$ that extend through more than one decade, reflecting the occurence of thermal events with no characteristic amplitude and time scales. A log-log fit to the distributions results in exponents $\alpha=3.2 \pm 0.8$ for the amplitudes and $\tau=4.3 \pm 0.8$ for the durations.

The sequences of heat-flow pulses, $\Psi(t)$, have been used to calculate a time correlation function $c(t)$ defined as:

$$
c(t)=\frac{1}{\Delta} \int_{0}^{\Delta} \Psi(u) \Psi(t+u) d u-\left(\frac{1}{\Delta} \int_{0}^{\Delta} \Psi(u) d u\right)^{2},
$$

where it is assumed that $c(t) \sim 0$ for $t>\Delta$. We have chosen $\Delta=100 s$ for sequences extending over $5400 \mathrm{~s}$. The average of $c(t) / c(0)$ over 7 loading and 5 unloading sequences is shown in the inset to figure 2, while the figure itself displays the corresponding power spectrum $c(\nu)$ obtained as the Discrete Fourier Transform of the correlation function. The power spectrum follows a power-law of the form $c(\nu) \sim \nu^{-\phi}$ over more than one decade, with $\phi=0.87 \pm 0.1$.

Second, the thermally induced $\mathrm{MT}$ of a Cu-17 at \% $\mathrm{Zn}-13.7$ at \% $\mathrm{Al}$ single crystal has been studied using acoustic emission (AE) techniques [5,6]. The crystal has a nominal $M_{s} \sim 299 K$. The experimental setup consists of (i) a heating and cooling stage, that allows reproducible thermal cycles at rates from $0.1 \mathrm{~K} / \mathrm{min}$ to $8 \mathrm{~K} / \mathrm{min}$, and (ii) a chain for the amplification (60dB in the range $400 \mathrm{kHz}-2 \mathrm{MHz}$ ) and acquisition (sampling rate $0.5 \mathrm{MHz}$ ) of the $\mathrm{AE}$ pulses recorded by a piezoelectric transducer attached to the sample.

We have determined the amplitude and duration of each signal, up to a total of about $2 \cdot 10^{4}$ signals in blocks of 1000 , recorded in small temperature intervals $(\sim 0.5 K)$ centered at five different temperatures in the transformation range. Our results indicate that the statistics of amplitudes $(A)$ and durations $(T)$ of the acoustic signals are nearly independent of the temperature around which they have been recorded. Moreover, the distributions obtained when all the signals recorded are considered together follow power-laws again, with $\alpha=3.8 \pm 0.8$ and $\tau=3.6 \pm 0.8$ for cooling, and $\alpha=3.5 \pm 0.8$ and $\tau=3.5 \pm 0.8$ for heating. Actually, the experimental uncertainties make the results for heating and cooling comparable. From the values of $\alpha$ and $\tau$ we can evaluate the exponent $x$ characteryzing the statistical relation $A \sim T^{x}$, making use [6] of the scaling relation $x(\alpha-1)=(\tau-1)$. The result, $x=0.9$, is consistent with the behaviour of the maximum of the joint statistical distribution of amplitudes vs. durations obtained experimentally. Finally, the power 

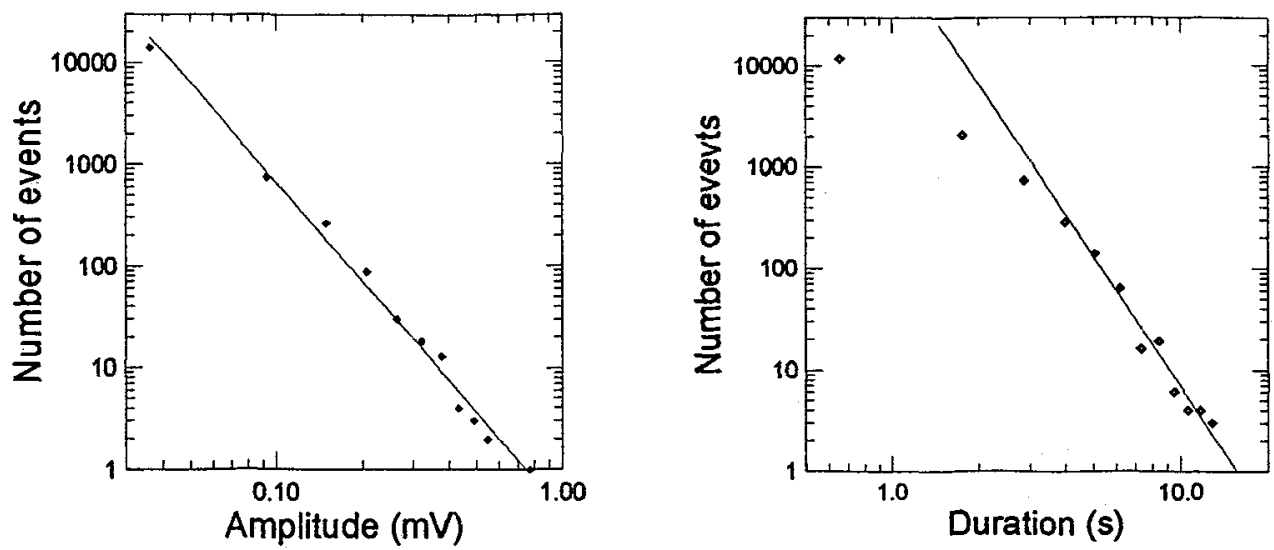

Figure 1. Histograms of amplitudes and durations of the heat pulses recorded during the stress-induced MT of a CuZnAl single crystal. Data is from 7 loading and 5 unloading tests. The straight lines are power-law fits with exponents $\alpha=3.2$ for the amplitudes and $\tau=4.3$ for the durations.

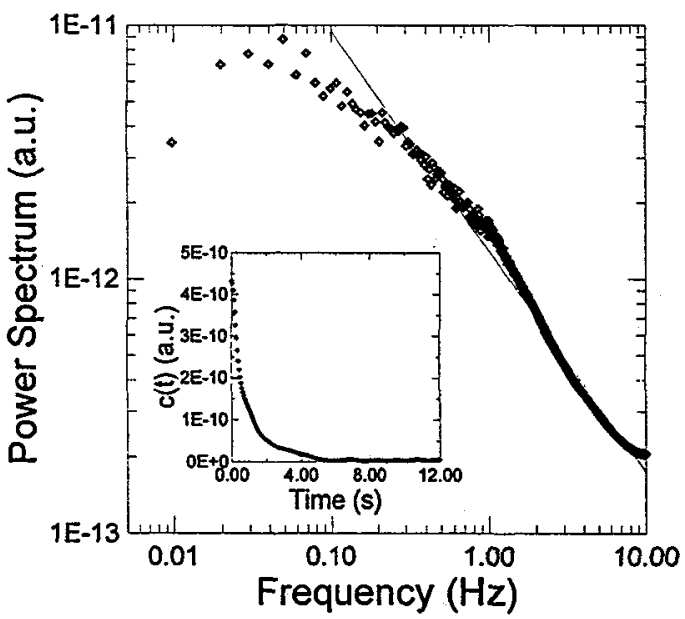

Figure 2. Correlation function (inset) and power spectrum computed for the sequences of heat pulses analyzed in figure 1 . The line is a power-law fit with exponent $\phi=0.87$.

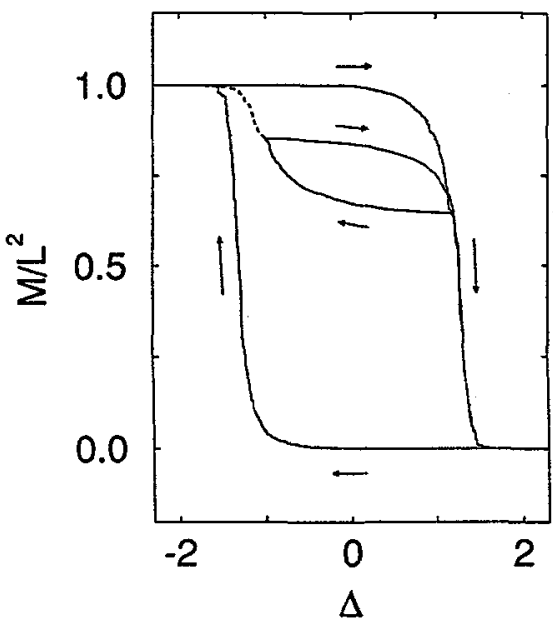

Figure 3. Hysteresis cycle of a $2 \mathrm{~d}$ RFBEG model of size $100 \times 100$, driven by the field $\Delta$ at $H=0$. Here $\sigma=1.3$ $\left(>\sigma_{c}(L)\right)$.

spectrum has also been found to follow a power-law, with an exponent $\phi=0.9 \pm 0.1$.

\section{MODELS}

This section analyzes several spin models with frozen disorder, defined on a $d$-dimensional square lattice of linear size $L$, and simulated at $T=0$. Their generic Hamiltonian reads:

$$
\mathcal{H}=-\sum_{\langle i, j\rangle} S_{i} S_{j}-K \sum_{\langle i, j\rangle} S_{i}^{2} S_{j}^{2}-H \sum_{i} S_{i}-\Delta \sum_{i} S_{i}^{2}+\mathcal{H}_{D}
$$

where $S_{i}$ is a generic spin variable, $K$ an interaction constant, $H$ an external field acting on the $S_{i}, \Delta$ a second external field acting on the $S_{i}^{2}$, and $\mathcal{H}_{D}$ a term containing the frozen disorder. The sum between 
$<>$ extends over all nearest neighbours pairs.

The first set of models of interest here are the Blume-Emery-Griffiths (BEG) models with disorder [7], in which the spin variables can take the three values $\pm 1,0$. Among the possible disorder terms, we have only considered $\mathcal{H}_{\bar{D}}^{\Delta}=\sum_{i} \Delta_{i} S_{i}^{2}$ where, for simplicity, the static field $\Delta_{i}$ follows a random gaussian distribution with zero mean and standard deviation $\sigma_{\Delta}$.

This model is well suited to describe a first-order phase transition between a single phase (all $\left.S_{i}=0\right)$ and a degenerated phase $\left(S_{i}= \pm 1\right)$, reminiscent of a thermally-induced MT between a $\beta$-phase single crystal and two symmetry-related martensite variants. The fields $H$ and $\Delta$ play different roles. $\Delta$ would be a temperature in the MT: $\Delta>0$ favours $S_{i}=0$, while $\Delta<0$ favours $S_{i}= \pm 1$ (without distinction). $H$ would be a uniaxial stress in the MT: $H>0$ favours $S_{i}=1$ and $H<0$ favours $S_{i}=-1$, i.e $H$ breaks the degeneracy of the \pm 1 phase. The order parameters conjugate to the two fields are $M=\sum_{i} S_{i}^{2}$ (the transformed fraction in the MT) and $m=\sum_{i} S_{i}$ (a. $2 d$-strain in the MT).

A second family is formed by the ferromagnetic Ising models with disorder. Now the $S_{i}$ can only take values \pm 1 , and hence the second and fourth terms of $\mathcal{H}$ can be ignored. $H$ is now the only driving field and $m=\sum_{i} S_{i}$ the only order parameter. At $T=0$, for large $H$, the +1 phase is stabilized. Upon decreasing the field, a pure relaxational dynamics (acceptance of all spin flips decreasing their local energy) drives the system along a sequence of metastable two-phase states connected by avalanches of spin flips until, for $H$ small enough, the pure -1 phase is reached. The precise behaviour of the system will depend on the nature of the frozen disorder. We have considered two kinds of disorder: random bonds (non symmetrybreaking terms) [8] such as $\mathcal{H}_{D}^{J}=\sum_{i, j} J_{i j} S_{i} S_{j}$, and random fields (symmetry-breaking terms) [9] such as $\mathcal{H}_{D}^{H}=\sum_{i} H_{i} S_{i}$. For simplicity, $J_{i j}$ and $H_{i}$ have been chosen again to follow gaussian distributions, with zero mean and standard deviation $\sigma_{J}$ and $\sigma_{H}$ respectively.

This second family of models is particularly well suited to reproduce a first-order phase transition between two symmetric phases, driven by an external field that breaks the degeneracy. For example, in the MT, the transition from one variant to another on going from uniaxial tension to uniaxial compression at constant temperature; or the reorientation of magnetic domains upon reversal of the external magnetic field in a ferromagnetic material.

In order to simulate the models we have used a synchronous dynamics (all unstable spins are updated simultaneously), which allows to define independently the size (magnitude of the jump in either $m$ or $M$, depending on which field is driving the system), and duration (number of updating steps) for each avalanche.

Figure 3 presents the hysteresis loop followed by the order parameter $M$ when a $2 d$ random-field BEG model (RFBEG) is cycled between extreme values of $\Delta$. Here we keep $H=0$ and $K=1$. For $\Delta \gg 0$ all spins in the system are in the state $S_{i}=0$, while for $\Delta \ll 0$ the spins can be found in one of the two states $S_{i}= \pm 1$, depending on their local random field and on the history of the system. Both forward and reverse evolutions take place by avalanches joining consecutive metastable states. Nevertheless, it is worth noting that the reverse path (increasing $\Delta$ ) does not correspond to the symmetrical of the forward one (decreasing $\Delta$ ), and that the internal trajectories tend to display Return-Point Memory [9,10], though not exactly because of the degeneracy of the $S_{i}= \pm 1$ phase with respect to $\Delta$.

To understand the influence of the disorder frozen in the system (parametrized by $\sigma$ ) upon the properties of the transformation cycle, we have performed a systematic analysis of the $H$-induced transition in the random bond (RBIM) and random field (RFIM) Ising models in $d=2$ and $d=3$, and of the $\Delta$-induced transition at $H=0$ in the RFBEG with disorder term $\mathcal{H}_{\vec{D}}^{\Delta}, K=1$ and $d=2$. For each model we have studied the statistical distribution of sizes and durations of the transformation avalanches.

Figure 4 presents the distributions obtained for excursions of increasing $\Delta$ in the RFBEG model, for three different amounts of frozen disorder parametrized by $\sigma_{\Delta}$. For small amounts of disorder (small $\sigma_{\Delta}$ ) the value of the random field is very similar in many lattice sites and hence a spin flip in one site is correlated to spin flips in many other sites, leading to a transformation dominated by very large avalanches; conversely, for a large amount of disorder the transformation events are practically uncorrelated (most of the spins change because of their own random field, independently of their neighbours) and the avalanches are predominantly small. There is a critical value of the disorder $\sigma_{c}$, however, at which avalanches of all sizes and durations develop (limited only by the size of the system) and the distributions become power-laws.

This change in the statistical properties of the transformation avalanches is acknowledged to be, on its 
Table 1: Critical exponents from zero temperature simulations of the disorder induced phase transition for the RFIM, the RBIM and the RFBEG in $2 d$ and $3 d$.

\begin{tabular}{lllll}
\hline \hline \multirow{2}{*}{ Model } & \multicolumn{2}{l}{ Exponent of the avalanche size distribution } & \multicolumn{2}{l}{ Critical value of disorder $\sigma_{c}$} \\
\cline { 2 - 6 } & $2 d$ & $3 d$ & $2 d$ & $.3 d$ \\
\hline RFIM & $1.3 \pm 0.2$ & $1.8 \pm 0.1$ & $0.75 \pm 0.03$ & $2.18 \pm 0.08$ \\
RBIM & $1.45 \pm 0.1$ & $2.0 \pm 0.2$ & $0.44 \pm 0.03$ & $1.12 \pm 0.05$ \\
RFBEG-DOWN & $1.3 \pm 0.1$ & & $0.51 \pm 0.03$ & \\
REFBEG-UP & $1.5 \pm 0.1$ & $0.42 \pm 0.02$ \\
\hline \hline
\end{tabular}

turn, a non-equilibrium phase transition mediated by the amount of disorder frozen in the system [8,9]. We have computed the critical exponents of this transition for the three models described above, using finite-size scaling techniques. Details can be found in ref. [11]. It is remarkable that the values obtained are consistent with a universal behaviour depending only on the space dimensionality $d$. Some of the results are presented in Table 1.
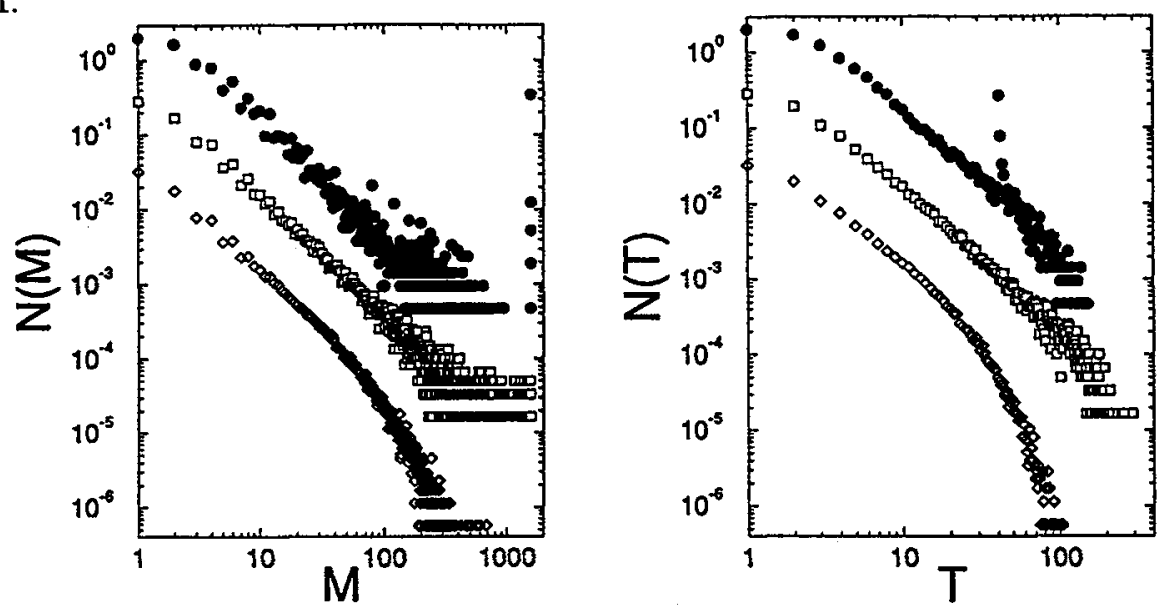

Figure 4. Distributions of avalanche sizes and durations in a 2d-RFBEG model driven by increasing $\Delta$ at $H=0$. The system size is $40 \times 40$. The data has been obtained from statistical averages over 1000 realizations of the random fields $\left\{\Delta_{i}\right\}$ with typical deviations $\sigma_{\Delta}=0.30$ (dots), 1.10 (squares) (close to the critical $\sigma_{\Delta}$ for this system size), and 2.00 (diamonds).

\section{DISCUSSION AND CONCLUSIONS}

We have regarded the thermoelastic MT as a dynamic process in a complex dissipative system with many degrees of freedom. Since the transformation is athermal (i.e. thermal fluctuations have a negligible probability of being large enough to overcome the energy barriers that separate the different metastable two-phase states available to the system along the MT path), it must be driven by an external field. In this scenario the evolution takes place by avalanches, which do not exhibit a characteristic size or duration.

Our experiments have shown that, remarkably, the statistical features of sizes and durations of the avalanches do not depend upon the geometry of the microstructures formed in the MT, the degeneracy of the low temperature phase or the kind of driving. Power-laws with the same exponents (within experimental uncertainty) have been found in both thermally and stress-induced transitions. This points to the idea that these features originate directly from (i) the complex elastic strain field resulting from transformation strain and (ii) from defects, frozen in the system or produced by the transformation. 
We have analyzed a series of simple models, which share the following characteristics with the systems of interest: they experience a first-order phase transition in which (given that simulations are carried out at $T=0$ ) thermal fluctuations are irrelevant; the transition is driven by external fields that either break or don't break the degeneracy of the low temperature phase; all of them incorporate frozen disorder, essential to their kinetic behaviour. The models give rise to hysteresis transformation cycles noticeably similar to experimental ones. In particular (although, for conciseness, we have not dealt with these questions), the origin of the intriguing Return-Point Memory and the influence of the defect concentration on the shape and properties of the hysteresis cycle can be exactly understood on the light of the models [7-9]. These models, at the critical disorder concentration, show universal properties depending only on dimensionality. Their statistical distributions of sizes and durations of avalanches, however, follow exact power-laws only at this given (critical) disorder concentration. It is difficult to understand how an actual system would spontaneously settle at such a particular disorder concentration. It seems much more plausible that, given that the critical region for the models is very large and that experiments on the statistics of avalanches are seriously limited in the number of decades of sizes and durations that can be confidently explored, real systems are simply close to but not quite at the critical disorder concentration.

Finally, we want to point out that the numerical values of the exponents obtained experimentally in the MT and the values of the exponents resulting from computer simulations of the spin models must be compared with caution, since (i) amplitudes of the MT events measured experimentally cannot be straightforwardly related to sizes of spin avalanches and (ii) complex elastic domain-domain and domain-defects interactions in MT systems are replaced in the models by simple short-range spin interactions with random couplings and random local fields.

\section{Acknowledgements}

This work has received financial support from the CICyT, project nr. MAT92-884.

\section{References}

[1] Bak P., Tang C., Wiesenfeld K., Phys. Rev. Lett. 59 (1987) 381; Phys. Rev. A 38 (1988) 364.

[2] Sornette D., J. de Physique 4 (1994) 209.

[3] Babcock K.L., Westervelt R.M., Phys. Rev. Lett. 64 (1990) 2168; Cote P.J., Meisel L.V., Phys. Rev. Lett. 91 (1991) 1334; Lilly M.P., Finley P.T., Hallock R.B., Phys. Rev. Lett. 71 (1993) 4186; Cannelli G., Cantelli R., Cordero F., Phys. Rev. Lett. 70 (1993) 3923; Wu W., Adams P.W., Phys. Rev. Lett. 74 (1995) 610.

[4] Khachaturyan A.G., Theory of Structural transformations in Solids (Wiley, New York, 1983).

[5] Vives E., Ortín J., Mañosa Ll., Ràfols I., Pérez-Magrané R., Planes A., Phys. Rev. Lett. 72 (1994) 1694; Vives E., Ortín J., Mañosa Ll., Ràfols I., Planes A., J. de Physique IV 5 (1995) C2-59; Vives E., Ràfols I., Mañosa Ll., Ortín J., Planes A., Phys. Rev. B (1995), submitted.

[6] Ràfols I., Vives E., Phys. Rev. B (1995), submitted.

[7] Goicoechea J., Ortín J., J. de Physique IV 5 (1995) C2-71.

[8] Vives E., Planes A., Phys. Rev. B 50 (1994) 3839.

[9] Sethna J.P., Dahmen K., Kartha S., Krumhansl J.A., Roberts B.W., Shore J.D., Phys. Rev. Lett. 70 (1993) 3347; Dahmen K., Sethna J.P., Phys. Rev. Lett. 17 (1993) 3222; Sethna J.P., Dahmen K., Kartha S., Krumhansl J.A., Perkovic O., Roberts B.W., Shore J.D., Phys. Rev. Lett. 72 (1994) 947.

[10] Ortín J., J. Appl. Phys. 71 (1992) 1454, and references therein.

[11] Vives E., Goicoechea J., Ortín J., Planes A., Phys. Rev. E (July 1995), in press. 